\title{
First order chemical reaction and thermal radiation effects on unsteady flow past an accelerated isothermal infinite vertical plate
}

\author{
R. Muthucumaraswamy ${ }^{1}$ and M. Ravi Shankar ${ }^{2}$ \\ ${ }^{1}$ Department of Applied Mathematics, ${ }^{2}$ Department of Chemical Engineering, Sri Venkateswara College of \\ Engineering, Sriperumbudur-602105, India. \\ msamy@svce.ac.in
}

\begin{abstract}
Theoretical solution of unsteady radiative flow past a uniformly accelerated isothermal infinite vertical plate with uniform mass diffusion is presented here, taking in to account the homogeneous chemical reaction of first order. The plate temperature is raised to $T_{w}$ and the concentration level near the plate is also raised to $C_{w}^{\prime}$. The dimensionless governing equations are solved using Laplace-transform technique. The velocity, temperature and concentration fields are studied for different physical parameters like thermal Grashof number, mass Grashof number, Schmidt number, Prandtl number, radiation parameter, chemical reaction parameter and time. It is observed that the velocity increases with increasing values of thermal Grashof number or mass Grashof number. But the trend is just reversed with respect to the thermal radiation parameter. It is also observed that the velocity increases with decreasing chemical reaction parameter.
\end{abstract}

Keywords: Accelerated; isothermal; vertical plate; chemical reaction; radiation.

Introduction

Chemical reactions can be codified as either heterogeneous or homogeneous processes. This depends on whether they occur at an interface or as a single phase volume reaction. In well-mixed systems, the reaction is heterogeneous, if it takes place at an interface and homogeneous, if it takes place in solution. In most cases of chemical reactions, the reaction rate depends on the concentration of the species itself. A reaction is said to be of first order, if the rate of reaction is directly proportional to the concentration itself. Chambre and Young (1958) have analyzed a first order chemical reaction in the neighborhood of a horizontal plate. Das et al. (1994) have studied the effect of homogeneous first order chemical reaction on the flow past an impulsively started infinite vertical plate with uniform heat flux and mass transfer. Again, mass transfer effects on moving isothermal vertical plate in the presence of chemical reaction studied by Das et al. (1999). The dimensionless governing equations were solved by the usual Laplacetransform technique.

Radiative heat and mass transfer play an important role in manufacturing industries for the design of fins, steel rolling, nuclear power plants, gas turbines and various propulsion device for aircraft, combustion and furnace design, materials processing, energy utilization, temperature measurements, remote sensing for astronomy and space exploration, food processing and cryogenic engineering, as well as numerous agricultural, health and military applications. England and Emery (1969) have studied the thermal radiation effects of an optically thin gray gas bounded by a stationary vertical plate. Radiation effect on mixed convection along a isothermal vertical plate were studied by Hossain and
Takhar (1996). Raptis and Perdikis (1999) studied the effects of thermal radiation and free convection flow past a moving vertical plate. The governing equations were solved analytically. Das et al. (1996)[5] have analyzed radiation effects on flow past an impulsively started infinite isothermal vertical plate. The dimensionless governing equations were solved by the usual Laplacetransform technique.

Gupta et al (1979) studied free convection on flow past a linearly accelerated vertical plate in the presence of viscous dissipative heat using perturbation method. Kafousias and Raptis (1981) extended the above problem to include mass transfer effects subjected to variable suction or injection. Free convection effects on flow past an exponentially accelerated vertical plate was studied by Singh and Naveen Kumar (1984). The skin friction for accelerated vertical plate has been studied analytically by Hossain and Shayo (1986). Mass transfer effects on flow past a uniformly accelerated vertical plate was studied by Soundalgekar (1982). Again, mass transfer effects on flow past an accelerated vertical plate with uniform heat flux was analyzed by Singh and Singh (1983). Basant Kumar Jha and Ravindra Prasad (1991) analyzed mass transfer effects on the flow past an accelerated infinite vertical plate with heat sources.

Hence, it is proposed to study the first order chemical reaction on unsteady flow past a uniformly accelerated isothermal infinite vertical plate with heat and mass transfer, in the presence of thermal radiation The dimensionless governing equations are solved using the Laplace-transform technique. The solutions are in terms of exponential and complementary error function. Such a study found useful in chemical process industries such as
Research article

CIndian Society for Education and Environment (iSee)
"Radiative heat \& Mass transfer" http://www.indjst.org
Muthucumaraswamy \& Ravi Shankar. Indian J.Sci.Technol. 
wire drawing, fibre drawing, food processing and polymer production.

\section{Mathematical formulation}

Here the unsteady radiative flow of a viscous incompressible fluid past a uniformly accelerated isothermal infinite vertical plate with uniform mass diffusion in the presence of chemical reaction of first order has been considered. Here the unsteady flow of a viscous incompressible fluid which is initially at rest and surrounds an infinite vertical plate with temperature $T_{\infty}$ and concentration $C_{\infty}^{\prime}$. The $x$-axis is taken along the plate in the vertically upward direction and the $y$-axis is taken normal to the plate. At time $t^{\prime} \leq 0$, the plate and fluid are at the same temperature $T_{\infty}$. At time $t^{\prime}>0$, the plate is accelerated with a velocity $u=\frac{u_{0}^{3} t^{\prime}}{v}$ in its own plane and the temperature from the plate is raised to $T_{w}$ and the concentration levels near the plate are also raised to $C_{w}^{\prime}$. It is assumed that the effect of viscous dissipation is negligible in the energy equation and there is a first order chemical reaction between the diffusing species and the fluid. The fluid considered here is a gray, absorbingemitting radiation but a non-scattering medium. Then under usual Boussinesq's approximation the unsteady flow is governed by the following equations:

$$
\begin{aligned}
& \frac{\partial u}{\partial t^{\prime}}=g \beta\left(T-T_{\infty}\right)+g \beta^{*}\left(C^{\prime}-C_{\infty}^{\prime}\right)+v \frac{\partial^{2} u}{\partial y^{2}} \\
& \rho C_{p} \frac{\partial T}{\partial t^{\prime}}=k \frac{\partial^{2} T}{\partial y^{2}}-\frac{\partial q_{r}}{\partial y} \\
& \frac{\partial C^{\prime}}{\partial t^{\prime}}=D \frac{\partial^{2} C^{\prime}}{\partial y^{2}}-K_{l}\left(C^{\prime}-C_{\infty}^{\prime}\right)
\end{aligned}
$$

With the following initial and boundary conditions:

$$
\begin{aligned}
& u=0, \quad T=T_{\infty}, \quad C^{\prime}=C_{\infty}^{\prime} \quad \text { for all } y, t^{\prime} \leq 0 \\
& t^{\prime}>0: \quad u=\frac{u_{0}^{3} t^{\prime}}{v}, \quad T=T_{w}, \quad C^{\prime}=C_{w}^{\prime} \\
& \text { at } y=0 \quad(4) \\
& u \rightarrow 0, \quad T \rightarrow T_{\infty}, \quad C^{\prime} \rightarrow C_{\infty}^{\prime} \quad \text { as } y \rightarrow \infty
\end{aligned}
$$

The local radiant for the case of an optically thin gray gas is expressed by $\frac{\partial q_{r}}{\partial y}=-4 a^{*} \sigma\left(T_{\infty}^{4}-T^{4}\right)$

It is assume that the temperature differences within the flow are sufficiently small such that $T^{4}$ may be expressed as a linear function of the temperature. This is accomplished by expanding $T^{4}$ in a Taylor series about $T_{\infty}$ and neglecting higher-order terms, thus

$T^{4} \cong 4 T_{\infty}^{3} T-3 T_{\infty}^{4}$

By using equations (5) and (6), equation (2) reduces to

$$
\rho C_{p} \frac{\partial T}{\partial t^{\prime}}=k \frac{\partial^{2} T}{\partial y^{2}}+16 a^{*} \sigma T_{\infty}^{3}\left(T_{\infty}-T\right)
$$

On introducing the following non-dimensional quantities:

$$
\begin{aligned}
& U=\frac{u}{u_{0}}, t=\frac{t^{\prime} u_{0}^{2}}{v}, Y=\frac{y u_{0}}{v}, \theta=\frac{T-T_{\infty}}{T_{w}-T_{\infty}}, \\
& G r=\frac{g \beta v\left(T_{w}-T_{\infty}\right)}{u_{0}^{3}}, C=\frac{C^{\prime}-C_{\infty}^{\prime}}{C_{w}^{\prime}-C_{\infty}^{\prime}}, G c=\frac{v g \beta^{*}\left(C_{w}^{\prime}-C_{\infty}^{\prime}\right)}{u_{0}^{3}}, \\
& R=\frac{16 a^{*} v^{2} \sigma T_{\infty}^{3}}{k u_{0}^{2}}, \operatorname{Pr}=\frac{\mu C_{p}}{k}, S c=\frac{v}{D}, K=\frac{v K_{l}}{u_{0}^{2}}
\end{aligned}
$$

in equations (1), (3) and (7) reduces to

$$
\begin{aligned}
& \frac{\partial U}{\partial t}=G r \theta+G C C+\frac{\partial^{2} U}{\partial Y^{2}} \\
& \frac{\partial \theta}{\partial t}=\frac{1}{\operatorname{Pr}} \frac{\partial^{2} \theta}{\partial Y^{2}}-\frac{R}{\operatorname{Pr}} \theta
\end{aligned}
$$

$\frac{\partial C}{\partial t}=\frac{1}{S c} \frac{\partial^{2} C}{\partial Y^{2}}-K C$

The negative sign of $\mathrm{K}$ in the last term of the equation (11) indicates that the chemical reaction takes place from higher level of concentration to lower level of concentration.

The initial and boundary conditions in non-dimensional quantities are

$$
\begin{aligned}
& U=0, \quad \theta=0, \quad C=0 \quad \text { for all } \quad Y, t \leq 0 \\
& t>0: \quad U=t, \quad \theta=1, \quad C=1 \quad \text { at } \quad Y=0 \\
& U \rightarrow 0, \quad \theta \rightarrow 0, \quad C \rightarrow 0 \quad \text { as } \quad Y \rightarrow \infty
\end{aligned}
$$


Vol. 4 No. 5 (May 2011)
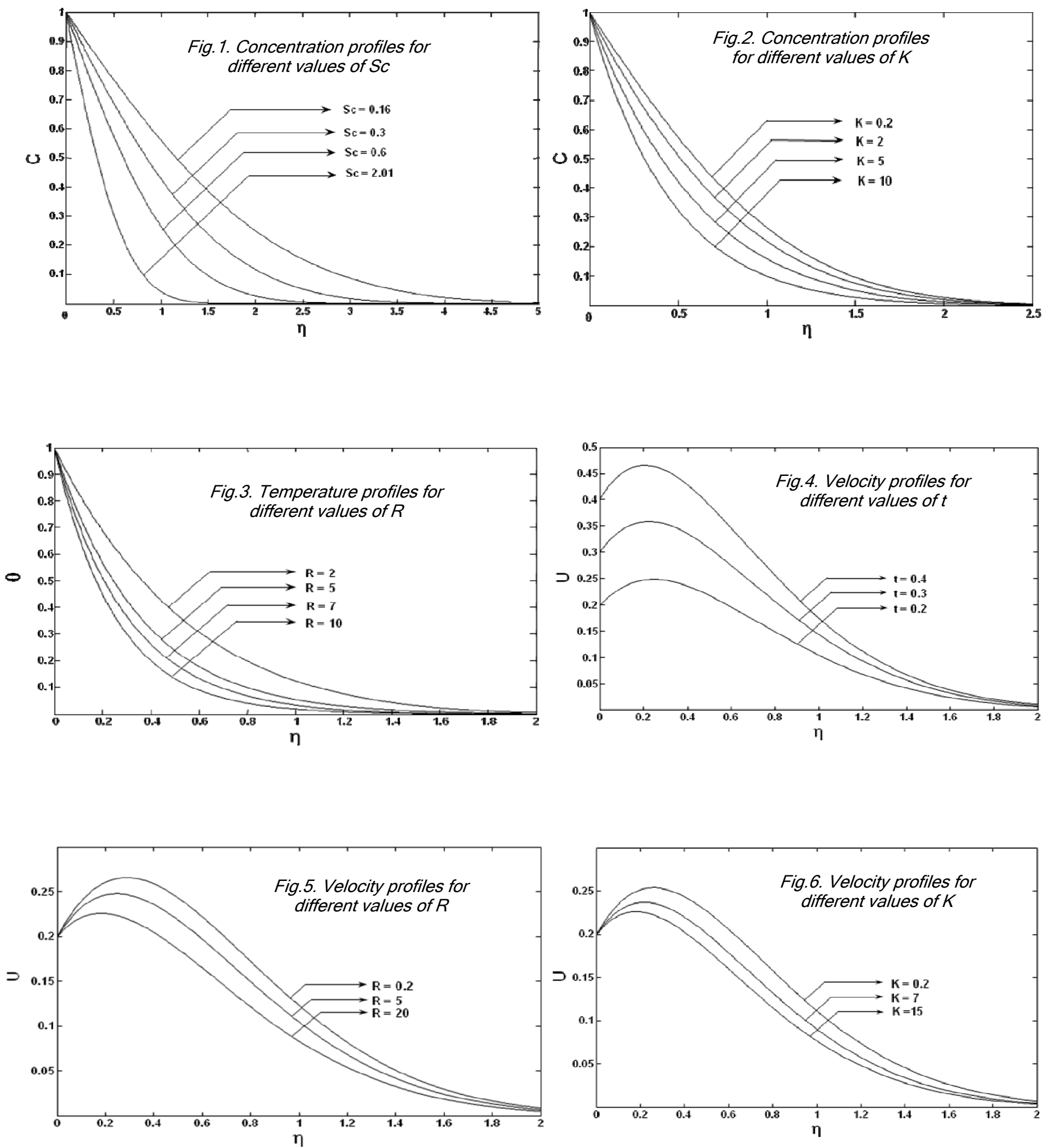
The dimensionless governing equations (9) to (11), subject to the initial and boundary conditions (12), are solved by the usual Laplace-transform technique and the solutions are derived as follows:

$$
\begin{aligned}
& \theta=\frac{1}{2}[\exp (2 \eta \sqrt{R t}) \operatorname{erf}(\eta \sqrt{\mathrm{Pr}}+\sqrt{a t})+\exp (-2 \eta \sqrt{R t}) \operatorname{erf}(\eta \sqrt{\mathrm{Pr}}-\sqrt{a t})] \\
& C=\frac{1}{2}[\exp (2 n \sqrt{K t S c}) \operatorname{erf}(r \sqrt{S c}+\sqrt{K t})+\exp (-2 r \sqrt{K t S c}) \operatorname{erf}(n \sqrt{S c}-\sqrt{K t})] \\
& U=t\left[\left(1+2 \eta^{2}\right) \cdot \operatorname{erfc}(\eta)-\frac{2 \eta}{\sqrt{\pi}} \exp \left(-\eta^{2}\right)\right]+2(d+e) \operatorname{erfc}(\eta) \\
& -d \exp (b t)[\exp (2 \eta \sqrt{b t}) \operatorname{erfc}(\eta+\sqrt{b t})+\exp (-2 \eta \sqrt{b t}) \operatorname{erfc}(\eta-\sqrt{b t})] \\
& -e \exp (c t)[\exp (2 \eta \sqrt{c t}) \operatorname{erfc}(\eta+\sqrt{c t})+\exp (-2 \eta \sqrt{c t}) \operatorname{erfc}(\eta-\sqrt{c t})]
\end{aligned}
$$

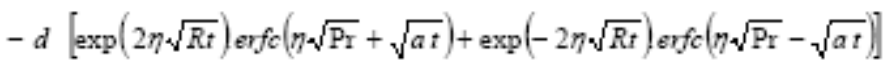

$$
\begin{aligned}
& +d \exp (b t)[\exp (-2 \eta \sqrt{\operatorname{Pr}(a+b) t}) \operatorname{erfc}(\eta \sqrt{\operatorname{Pr}}-\sqrt{(a+b) t}) \\
& +\exp (2 \eta \sqrt{\operatorname{Pr}(a+b) t}) \operatorname{erfc}(\eta \sqrt{\operatorname{Pr}}+\sqrt{(a+b) t})]
\end{aligned}
$$

$$
-e[\exp (-2 \eta \sqrt{K t S c}) \operatorname{erfc}(\eta \sqrt{S c}-\sqrt{K t})-\exp (2 \eta \sqrt{K t S c}) \operatorname{erfc}(\eta \sqrt{S c}+\sqrt{K t})]
$$$$
+e \exp (c t) \mid \exp (-2 \eta \sqrt{S c(K+c) t}) \operatorname{erfc}(\eta \sqrt{S c}-\sqrt{(K+c) t})
$$

$$
+\exp (2 \eta \sqrt{S c(K+c) t}) \operatorname{erfc}(\eta \sqrt{S c}+\sqrt{(K+c) t})]
$$

Where,

$a=\frac{R}{P r}, b=\frac{R}{1-P r}, c=\frac{K S c}{1-S c}, d=\frac{G r}{2 b(1-P r)}, e=\frac{G c}{2 c(1-S c)}$ and $\eta=\frac{Y}{2 \sqrt{t}}$ dominant in concentration field. It is observed that the wall concentration increases with decreasing values of the Schmidt number. The effect of concentration profiles for different values of the chemical reaction parameter $(K=0.2,2,5,10)$ and time $t=0.2$ are presented in Fig.2. The effect of the chemical reaction parameter is dominant in concentration field. The profiles have the common feature that the concentration decreases in a monotone fashion from the surface to a zero value far away in the free stream. It is observed that the wall concentration increases with decreasing values of the chemical reaction parameter.

The temperature profiles are calculated for different values of thermal radiation parameter $(R=$ $2,5,7,10)$ and time $t=0.4$ from Equation (13) and these are shown in Fig.3. in the presence of air. The effect of thermal radiation parameter is important in temperature profiles. It is observed that the temperature increases with decreasing radiation parameter.

The velocity profiles for different values of $(t=0.2,0.4,0,6), K=0.2$

$\mathrm{Gr}=\mathrm{Gc}=2$ are studied and presented in Fig.4. It is observed that the velocity increases with increasing values of the time $t$. The effect of velocity for different values of the radiation parameter $(R=0.2,5,20), G r=G c=2$ and $t=0.2$ are shown in Fig.5. The trend shows that the velocity increases with decreasing radiation

\section{Results and discussion}

For physical interpretation of the problem, the numerical computations are carried out for different physical parameters $G r, G c, S c, R, \operatorname{Pr}, K$ and $t$ upon the nature of the flow and transport. The value of the Schmidt number $S c$ is taken to be 0.6 which corresponds to water-vapor. Also, the value of Prandtl number $\operatorname{Pr}$ are chosen such that they represent air( $\operatorname{Pr}=0.71$ ). The numerical values of the velocity, temperature and concentration are computed for different physical parameters like Prandtl number, thermal Grashof number, mass Grashof number, chemical reaction parameter, radiation parameter, Schmidt number and time are studied graphically.

The concentration profiles for different values of the Schmidt number $(\mathrm{Sc}=0.16,0.3,0.6,2.01), \mathrm{K}=0.2$ and time $t=0.2$ are presented in Fig.1. The effect of the Schmidt number is Research article CIndian Society for Education and Environment (iSee)

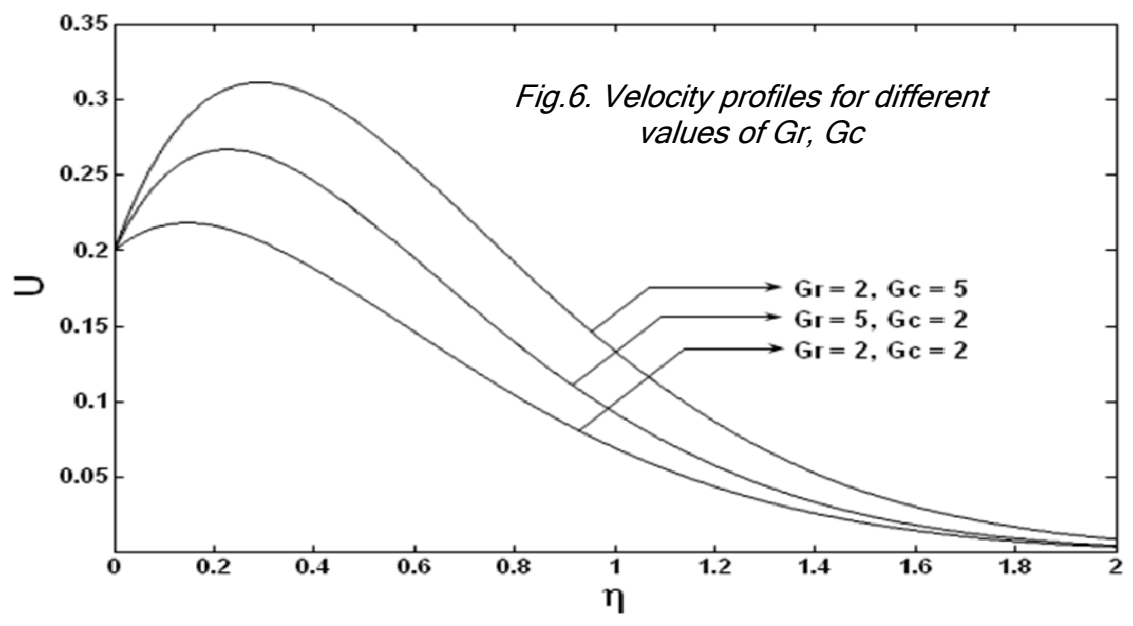

"Radiative heat \& Mass transfer" http://www.indjst.org parameter. It is observed that there is a fall in velocity in the presence of high thermal radiation.

Fig.6 illustrates the effect of velocity for different values of the chemical reaction parameter ( $K=0.2,7,15), G r=G c=2, R=5$ and $t=0.2$. This shows that the increase in the chemical reaction parameter leads to a fall in the velocity. Fig.7. demonstrates the effect velocity fields for different 
thermal Grashof number ( $G r=2,5$ ), mass Grashof number ( $G c=2,5), K=7, R=20$ and $t=0.2$. It is observed that the velocity increases with increasing values of the thermal Grashof number or mass Grashof number.

\section{Conclusion}

The theoretical solution of radiative flow past a uniformly accelerated isothermal infinite vertical plate with uniform mass diffusion, in the presence of homogeneous chemical reaction of first order has been studied. The dimensionless governing equations were solved by the usual Laplace-transform technique. The effect of different physical parameters like chemical reaction parameter, radiation parameter, thermal Grashof number, mass Grashof number and $t$ are studied graphically. It is observed that the velocity increases with increasing values of $G r, G c$ and $t$. But the trend is just reversed with respect to the chemical reaction parameter or radiation parameter.

\section{References}

1. Basant Kumar Jha, Ravindra Prasad and Surendra Rai (1991) Mass transfer effects on the flow past an exponentially accelerated vertical plate with constant heat flux. Astrophys. \& Space Sci. 181, 125-134.

2. Chambre PL. and Young JD (1958) On the diffusion of a chemically reactive species in a laminar boundary layer flow,. The Physics of Fluids. 1, 48-54.

3. Das U.N., Deka RK. and Soundalgekar VM (1994) Effects of mass transfer on flow past an impulsively started infinite vertical plate with constant heat flux and chemical reaction. Forschung im Ingenieurwesen. 60, 284-287.

4. Das U.N., Deka RK. and Soundalgekar VM (1999) Effects of mass transfer on flow past an impulsively started infinite vertical plate with chemical reaction. The Bull. of GUMA,. I.5 , 13-20.

5. Das UN, Deka RK and Soundalgekar VM (1996) Radiation effects on flow past an impulsively started vertical infinite plate. J. Theo. Mech. 1,111-115.

6. England WG and Emery AF (1969) Thermal radiation effects on the laminar free convection boundary layer of an absorbing gas. J. Heat Transfer. 91, 37-44.

7. Gupta AS, PoP I and Soudalgekar VM (1979) Free convection effects on the flow past an accelerated vertical plate in an incompressible dissipative fluid. Rev. Roum. Sci. Techn.- Mec. Apl. 24, 561-568.

8. Hossain MA and Shayo LK (1986) The skin friction in the unsteady free convection flow past an accelerated plate. Astrophys. \& Space Sci.125, 315324.

9. Hossain MA and Takhar HS (1996). Radiation effect on mixed convection along a vertical plate with
Vol. 4 No. 5 (May 2011)

ISSN: 0974- 6846

uniform surface temperature. Heat \& Mass Tr. 31, 243-248.

10.Kafousias NG and Raptis AA (1981) Mass transfer and free convection effects on the flow past an accelerated vertical infinite plate with variable suction or injection. Rev. Roum. Sci. Techn.-Mec. Apl. 26, 11-22.

11.Raptis A and Perdikis C (1999) Radiation and free convection flow past a moving plate. Int. J. Appl. Mech. Engg. 4, 817-821.

12.Singh AK and Naveen Kumar (1984) Free convection flow past an exponentially accelerated vertical plate. Astrophys. Space Sci. 98, 245-258.

13. Singh AK and Singh J (1983) Mass transfer effects on the flow past an accelerated vertical plate with constant heat flux. Astrophys. Space Sci. 97, 57-61.

14. Soundalgekar VM.(1982) Effects of mass transfer on flow past a uniformly accelerated vertical plate, Letters in Heat \& Mass Transfer. 9, 65-72.
"Radiative heat \& Mass transfer" http://www.indjst.org
Muthucumaraswamy \& Ravi Shankar. Indian J.Sci.Technol. 